\title{
Rare diffuse idiopathic pulmonary neuroendocrine cell hyperplasia: one case report and literature review
}

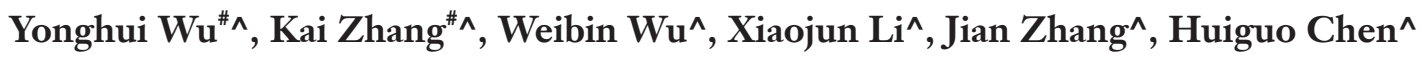 \\ Department of Cardiothoracic Surgery, the Third Affiliated Hospital of Sun Yat-sen University, Guangzhou, China \\ \#These authors contributed equally to this work. \\ Correspondence to: Huiguo Chen. Department of Cardiothoracic Surgery, the Third Affiliated Hospital of Sun Yat-sen University, No. 600 Tianhe \\ Road, Guangzhou 510630, China. Email: Andychen820727@hotmail.com.
}

\begin{abstract}
Rare diffuse idiopathic pulmonary neuroendocrine cell hyperplasia (DIPNECH) was first diagnosed in the early 1950s, but was not fully recognized and named until 1992. Literatures reported that DIPNECH usual was multiple and diffuse bilateral nodules, rare patients demonstrate the single nodule or ground glass nodule (GGN). We diagnosed one patient because of intermittent dry cough at least one year, Chest computed tomography (CT) found a purity GGN (pGGN) in the anterior segment, right upper lung lobe, and bronchoscopy didn't have any tumor in the bronchus in 2014. Continue follow-up 4 years, the nodule enlarged and became a mixed GGN (mGGN) in 2018 on chest CT. A diagnostics video-assisted thoracotomy with wedge resection in right upper lung lobe was performed. The pathology revealed that it was filled with neuroendocrine differentiation cells without penetration terminal bronchiole submucosal layer, immunohistochemical (IHC) staining were positive for CD-56, Cg-A, TTF, Syn and ki-67 (about 5\%), leading to diagnosis of DIPNECH. A regular review of the chest CT showed no signs of tumor recurrence postoperative more than 1 year every 6 months, and we will continue follow-up. In conclusion, DIPNECH keeps stable evolution over several years, which is misdiagnosed and underdiagnosed usually. Patient who showed GGN on chest CT and had cough, dyspnea, wheezing, less frequently hemoptysis and so on, we should think of DIPNECH. Diagnosis depended on pathology and IHC staining, regular follow-up will lead to patient a long-term survival postoperative.
\end{abstract}

Keywords: Ground glass nodule (GGN); neuroendocrine cells hyperplasia; immunohistochemical staining; case report

Submitted Apr 12, 2020. Accepted for publication Oct 30, 2020.

doi: $10.21037 /$ tcr-20-1783

View this article at: http://dx.doi.org/10.21037/tcr-20-1783

\section{Introduction}

DIPNECH as a rare lung disease and a premalignant lesion, characterized by a marked increase in neuroendocrine cells, it was reported by Aguayo above all (1). WHO histological classification revised in 2015, DIPNECH was added to be a precursor of tumorlets, which was less than $0.5 \mathrm{~cm}$ in diameter, and carcinoid tumors, which well-differentiated neuroendocrine cells proliferation had broken through epithelial basement membrane, and its diameter above $0.5 \mathrm{~cm}$ for carcinoid tumors $(2,3)$.

Because of bronchiolar fibrosis and chronic inflammation, DIPNECH demonstrates obstructive ventilatory pattern on lung function tests, most patients are usually misdiagnosed for asthma or bronchitis in the first. The treatments included in inhaled corticosteroids, systemic corticosteroids, bronchodilators, lung surgery and even lung

\footnotetext{
^ Yonghui Wu, ORCID: 0000-0003-2392-1382; Kai Zhang, ORCID: 0000-0003-3905-5095; Weibin Wu, ORCID: 0000-0002-2272-8735;

Xiaojun Li, ORCID: 0000-0001-7078-8241; Jian Zhang, ORCID: 0000-0001-5000-6142; Huiguo Chen, ORCID: 0000-0002-7877-4538.
} 

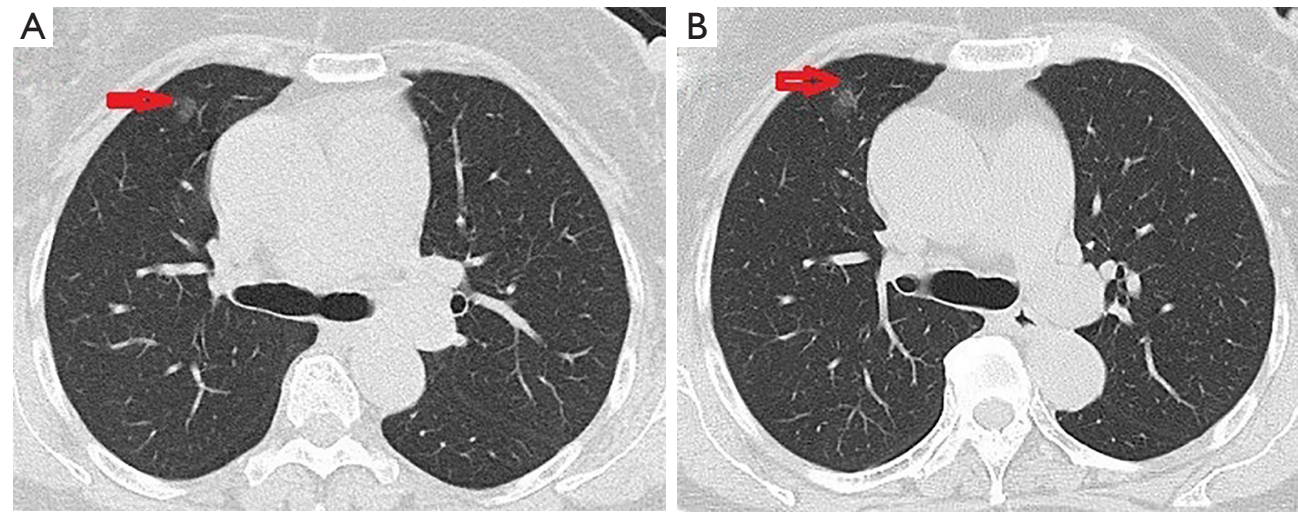

Figure 1 In 2014 chest CT clearly demonstrated a pGGN in the anterior segment, right upper lung lobe (A); in 2018, the nodule had enlarged and became an mGGN (B). The red arrows mark the lesion located in anterior segment, right upper lung lobe (A: pGGN; B: mGGN). pGGN, purity ground glass nodule; mGGN, mixed ground glass nodule.

transplantation (4). We manage a 72 -year-old, non-smoking woman who presented with right anterior segment lung nodule, was diagnosed DIPNECH by pathology and IHC staining. We present the following article in accordance with the CARE reporting checklist $(5,6)$ (available at http:// dx.doi.org/10.21037/tcr-20-1783).

\section{Case presentation}

All procedures performed in studies involving human participants were in accordance with the ethical standards of the institutional and/or national research committee(s) and with the Helsinki Declaration (as revised in 2013). Written informed consent was obtained from the patient.

A 72-year-old female visited our outpatient with intermittent dry cough at least one year and didn't associate with fever, expectoration, chest tightness and pain. She was a nonsmoker or passive smoker and had hypertension and diabetes several years. Chest CT showed a pGGN in the anterior segment, right upper lung lobe in 2014, the nodule maximum diameter is about $1.0 \mathrm{~cm}$, the derivation purified protein skin test and T-SPOT was negative, blood neuronspecific anolase (NSE) and carcinoembryonic antigen (CEA) level were normal, and bronchoscopy didn't have any tumor in the bronchus. Physical examination is normal and doesn't touch any enlarged lymph nodes on neck or supraclavicular, her lung function is normal.

Primary diagnosed pneumonia and after antibiotics treatment, dry cough recurred intermittent attack for 4 years. However, regular follow-up chest CT demonstrated that nodule didn't change every year. In 2018, nodule enlarged and became an mGGN on chest CT, maximum diameter is approximately $1.5 \mathrm{~cm}$, and the mGGN is diagnosed the primary lung carcinoma (Figure 1).

The NSE and CEA level, lung function was still normal. A diagnostics video-assisted thoracotomy with wedge resection in right upper lung lobe was performed. Pathological processing of specimen $1.5 \mathrm{~cm} \times 1.0 \mathrm{~cm}$ nodule revealed that it was filled with neuroendocrine differentiation cells and preformed IHC staining, the tumor was strongly positive for CD-56, Cg-A, TTF, Syn and ki-67 (about 5\%), leading to diagnosis of DIPNECH (Figure 2).

\section{Follow-up}

The patient wasn't performed any adjuvant treatments postoperative, and was managed by observation only and had been followed up more than 1 year. A regular review of the chest CT every 6 months showed no signs of tumor recurrence, dry cough and other complaints hadn't occurred, the blood NSE and CEA level, lung function was still almost normal.

A timeline showed the whole medical procedure of this case (Figure 3).

\section{Discussion}

DIPNECH is a rare and relatively new pulmonary condition, that is fully recognized in 1992, is confined to the respiratory epithelium layer without penetration of the basement membrane. The collection of cells which is smaller than $0.5 \mathrm{~cm}$ is called tumorlets, and the nodule 

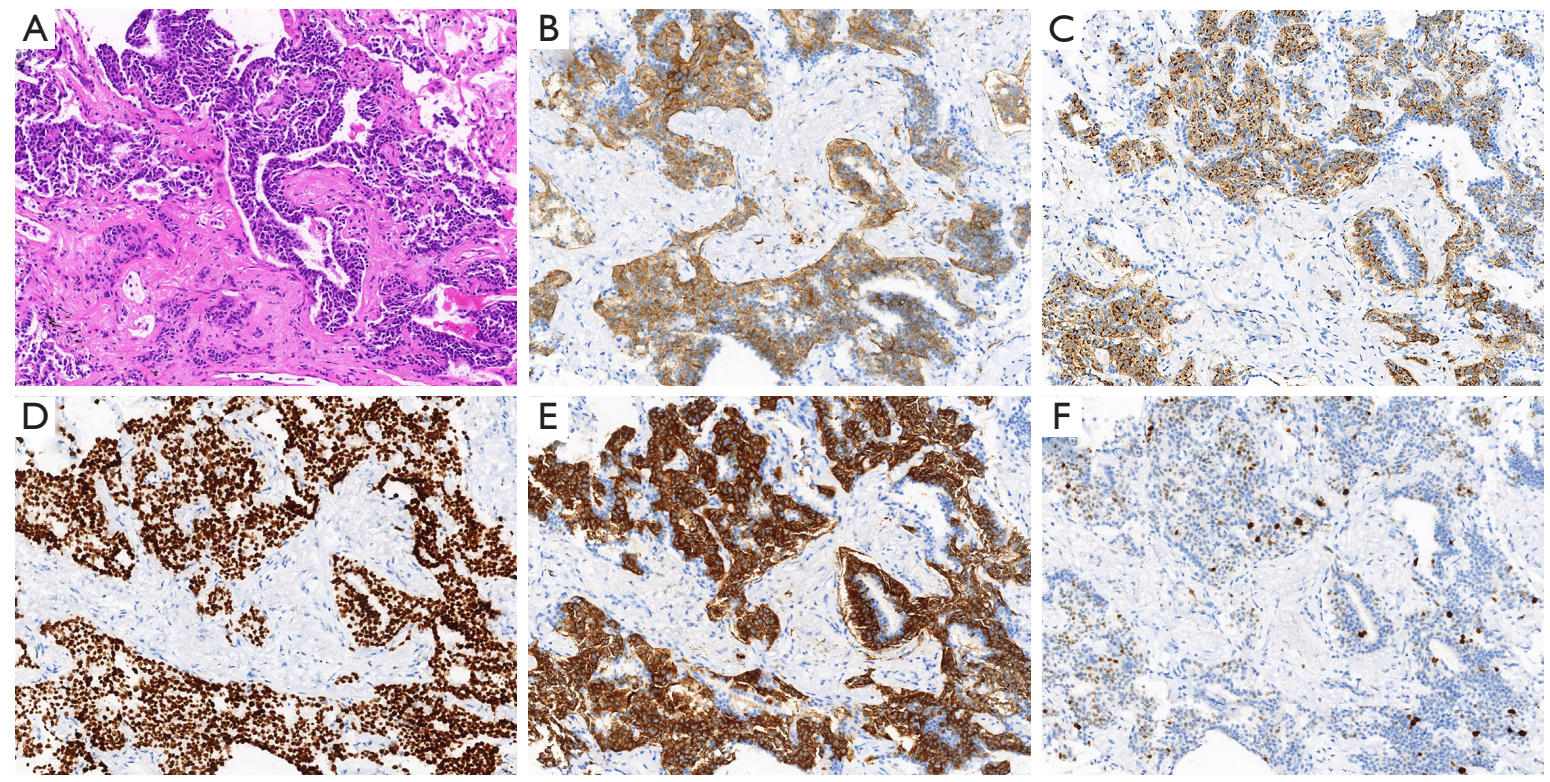

Figure 2 The nodule of proliferating neuroendocrine cells consistent with tumorlets [H\&E staining, ×200 (A)], it was characterized by spindle cells with round, oval with relatively same shape and size. It shows the number increase, less cytoplasm and large nuclei. Cell nuclei are stained deeply or nuclei are unobvious by $\mathrm{H} \& \mathrm{E}$ staining. Neuroendocrine cells were beyond the basement membrane and infiltrated with less than $0.5 \mathrm{~cm}$ diameter. IHC staining demonstrated that cells staining was positive for CD-56, $\times 200(\mathrm{~B}), \mathrm{Cg}-\mathrm{A}, \times 200(\mathrm{C}), \mathrm{TTF}, \times 200$ (D), Syn, $\times 200$ (E) and ki-67, ×200 (about 5\%) (F).

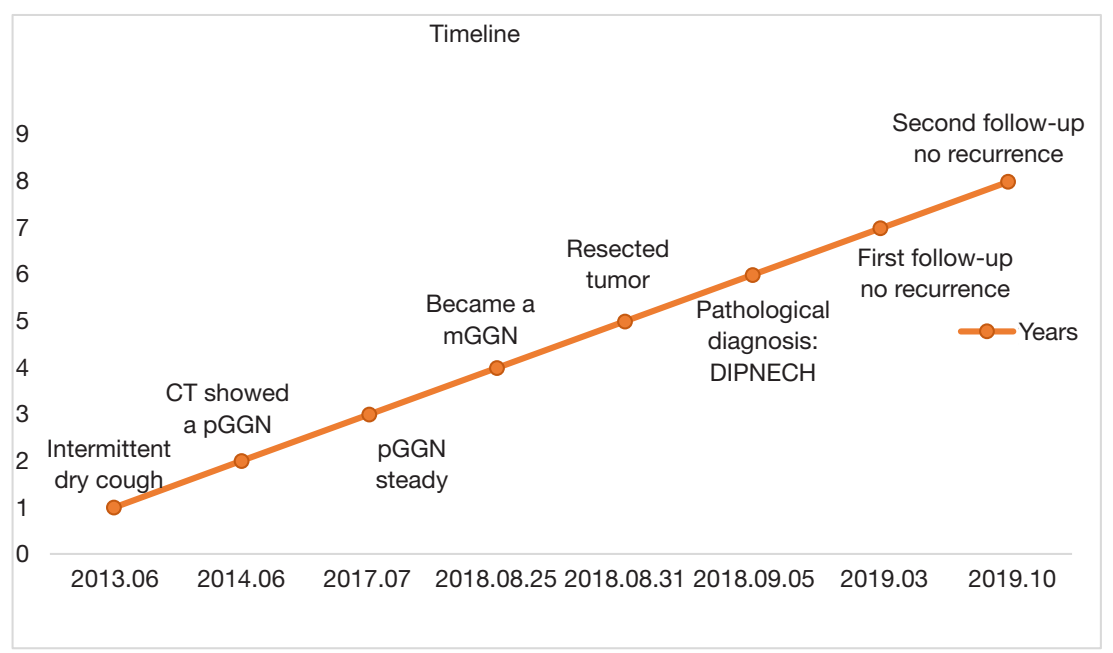

Figure 3 A timeline showing the whole medical procedure of this case. pGGN, purity ground glass nodule; mGGN, mixed ground glass nodule; DIPNECH, diffuse idiopathic pulmonary neuroendocrine cell hyperplasia.

greater than $0.5 \mathrm{~cm}$ is generally considered to be carcinoid tumor (7).

It is misdiagnosed for asthma or chronic bronchitis usually. Most patients are nonsmoker female between the ages of fifty and seventy with a dry cough and/or dyspnea, the clinical symptoms lowly worsens or keeps stable evolution over several years $(8,9)$. This patient is 68 years old at the first visit and regular follow-ups 4 years preoperative, dry cough symptom gradually worsens.

DIPNECH may be no clinical symptoms and is 
discovered accidentally, the physical examination even doesn't exist any positive signs. Pulmonary lesions are checked in radiological imaging. High-resolution CT and bronchoscopy are commended for diagnosing in the clinic. As the disease progresses, the pulmonary function test is diversity, that can be normal, obstructive, restrictive, or mixed obstructive restrictive dysfunction. This case, the lesion is local and lung function test is normal all the time. Neuroendocrine cells can release amines and peptides, which affect the respiratory tract, it is usually seen cough, dyspnea, and wheezing, even to asthma symptoms in patients (10).

DIPNECH usually manifests as bilateral and diffuse airway condition, air-trapping and pulmonary lesions in radiological imaging, only rare patient imaging demonstrate the single nodule or GGN, which isn't differentiated from primary lung carcinoma $(11,12)$. Multiple and diffuse pulmonary lesions usually are misdiagnosed as metastatic cancer. DIPNECH is typically rounded, noncalcified, and well-defined, which was differentiated from metastatic carcinoma.

DIPNECH is considered a precursor lesion for carcinoid tumors, and lesions follow a developmental course from DIPNECH to tumorlets and carcinoid. Diagnosis of DIPNECH need biopsy and an adequate tissue, pathological criteria present five or more NECs in tissue, either singly or in clusters (13). Firm the diagnosis of DIPNECH relied on pathology and IHC staining, endocrine markers Syn, CA-g and CD56 are all positive and CD10 is stained consistently in neuroendocrine cells. Otherwise bombesin, BCL-2, retinoblastoma protein, $\mathrm{p} 27$, and calcitonin staining is variable in diagnosis (8). In this case, IHC staining CD56, CA-g, TTF and Syn are positive and ki- 67 is only about $5 \%$, which is same to the literature reported.

The natural history of DIPNECH appears to be favorable for most patients experience stable and very slow progressive disease, and only a small number of patients lead to the severe diffuse small airway obstruction even to the respiratory failure, so conservative management is often used. Rare incidence, there are many controversies in DIPNECH treatment process. A "wait and watch" policy showed that the disease remained stable over many years $(14,15)$. Long-term surveillance is generally accepted to monitor for progression to this tumor. But if there is no clear diagnosis, radical surgical resection play a role in the local lesion, including in wedge resection, segmentectomy, lobectomy, a whole lung resection, and even to lung transplantation has been reported in patients with bilateral and diffuse nodules (10). The patient has followed up four years preoperative, the nodule gradually becomes large and entity. Postoperative the patient doesn't perform any adjuvant treatment and has followed up more than 1 year every 6 months, there isn't dry cough or any new complaints and no signs of tumor recurrence.

Some studies have showed that somatostatin analogs can alleviate the chronic cough, glucocorticoids are used the diffuse and advanced lesions (14). DIPNECH develops or combines associated carcinoid tumor, chemotherapy can prolong survival time and reduce recurrence for patients. However, lung transplantation has been performed in diffuse small airway obstruction or obliterative bronchiolitis.

However, there are several deficiencies in the whole course of treatment. First, we only report one case. Second, patient postoperative follow-up time is relatively short, and a long-term follow-up is very necessary to evaluate the therapeutic efficacy.

\section{Conclusions}

DIPNECH is a poorly understand and rare pulmonary disease, characterized by neuroendocrine cells hyperplasia in small airways or lung lobes. It usually affects nonsmoking female between the ages of fifty and seventy, the most common symptom is dry cough. It is checked in radiological imaging and need for differential diagnosis of asthma and bronchitis. Surgery play a role in the local lesion and firm the diagnosis depends on pathological and IHC staining. On account of very slow progression, a "wait and watch" policy perform the preoperative or postoperative for the most patients.

\section{Acknowledgments}

We would to thank Professor Luying Tang, Institute of Pathology, the Third Affiliated Hospital of Sun Yat-sen University, China, who provided the confirmation of the pathologic diagnosis of this case.

Funding: None.

\section{Footnote}

Reporting Checklist: The authors have completed the CARE reporting checklist. Available at http://dx.doi.org/10.21037/ tcr-20-1783

Conflicts of Interest: All authors have completed the ICMJE 
uniform disclosure form (available at http://dx.doi. org/10.21037/tcr-20-1783). The authors have no conflicts of interest to declare.

Ethical Statement: The authors are accountable for all aspects of the work in ensuring that questions related to the accuracy or integrity of any part of the work are appropriately investigated and resolved. The study was conducted in accordance with the Declaration of Helsinki (as revised in 2013). All procedures performed in studies involving human participants were in accordance with the ethical standards of the institutional and/or national research committee(s) and with the Helsinki Declaration (as revised in 2013). Written informed consent was obtained from the patient.

Open Access Statement: This is an Open Access article distributed in accordance with the Creative Commons Attribution-NonCommercial-NoDerivs 4.0 International License (CC BY-NC-ND 4.0), which permits the noncommercial replication and distribution of the article with the strict proviso that no changes or edits are made and the original work is properly cited (including links to both the formal publication through the relevant DOI and the license). See: https://creativecommons.org/licenses/by-nc-nd/4.0/.

\section{References}

1. Davies SJ, Gosney JR, Hansell DM, et al. Diffuse idiopathic pulmonary neuroendocrine cell hyperplasia: an under-recognised spectrum of disease. Thorax 2007:62:248-52.

2. Koliakos E, Thomopoulos T, Abbassi Z, et al. Diffuse Idiopathic Pulmonary Neuroendocrine Cell Hyperplasia: A Case Report and Review of the Literature. Am J Case Rep 2017:18:975-9.

3. Moonen L, Derks J, Dingemans AM. Orthopedia Homeobox (OTP) in Pulmonary Neuroendocrine Tumors: The Diagnostic Value and Possible Molecular Interactions. Cancers (Basel) 2019:11:1508.

4. Tippett VM, Wathen CG. Diffuse idiopathic neuroendocrine cell hyperplasia: an unusual cause of breathlessness and pulmonary nodules. BMJ Case Rep 2010;2010:bcr0520103006.

5. Werthmann PG, Saltzwedel G, Kienle GS. Minor regression and long-time survival (56 months) in a patient with malignant pleural mesothelioma under Viscum album and Helleborus niger extracts-a case report. J Thorac Dis 2017:9:E1064-70.

6. Gagnier JJ, Kienle G, Altman DG, et al. The CARE Guidelines: Consensus-based Clinical Case Reporting Guideline Development. Glob Adv Health Med 2013:2:38-43.

7. Cameron CM, Roberts F, Connell J. Diffuse idiopathic pulmonary neuroendocrine cell hyperplasia: an unusual cause of cyclical ectopic adrenocorticotrophic syndrome. The British Journal of Radiology 2011:84:e14-7.

8. Abrantes C, Oliveira RC, Saraiva J, et al. Pulmonary Peripheral Carcinoids after Diffuse Idiopathic Pulmonary Neuroendocrine Cell Hyperplasia and Tumorlets: Report of 3 Cases. Case Rep Pulmonol 2015:2015:851046.

9. Mandegaran R, David S, Screaton N. Cardiothoracic manifestations of neuroendocrine tumours. Br J Radiol 2016:89:20150787.

10. Cansız Ersöz C, Cangir AK, Dizbay Sak S. Diffuse Neuroendocrine Cell Hyperplasia: Report of Two Cases. Case Rep Pathol 2016:2016:3419725.

11. Jiramethee N, Erasmus D, Nogee L, et al. Pulmonary Neuroendocrine Cell Hyperplasia Associated with Surfactant Protein C Gene Mutation. Case Rep Pulmonol 2017:2017:9541419.

12. Jin L, Wang Z, Qi X. Diffuse idiopathic pulmonary neuroendocrine cell hyperplasia: Case series and a review of the literature. Medicine (Baltimore) 2018:97:e13806.

13. Nevel RJ, Garnett ET, Worrell JA, et al. Persistent Lung Disease in Adults with NKX2.1 Mutation and Familial Neuroendocrine Cell Hyperplasia of Infancy. Ann Am Thorac Soc 2016:13:1299-304.

14. Ofikwu G, Mani VR, Rajabalan A, et al. A rare case of diffuse idiopathic pulmonary neuroendocrine cell hyperplasia. Case Rep Surg 2015;2015:318175.

15. Takegahara K, Sato A, Ibi T, et al. Usuda J Atypical carcinoid localized at the bronchus accompanied by diffuse idiopathic pulmonary neuroendocrine cell hyperplasia in the distal lung: a rare case report. J Thorac Dis 2017:9:E774-8.

Cite this article as: $\mathrm{Wu} \mathrm{Y,} \mathrm{Zhang} \mathrm{K}, \mathrm{Wu} \mathrm{W}$, Li X, Zhang J, Chen $\mathrm{H}$. Rare diffuse idiopathic pulmonary neuroendocrine cell hyperplasia: one case report and literature review. Transl Cancer Res 2020;9(12):7657-7661. doi: 10.21037/tcr-20-1783 